\title{
Stroke treatment and prevention
}

\section{Five new things}

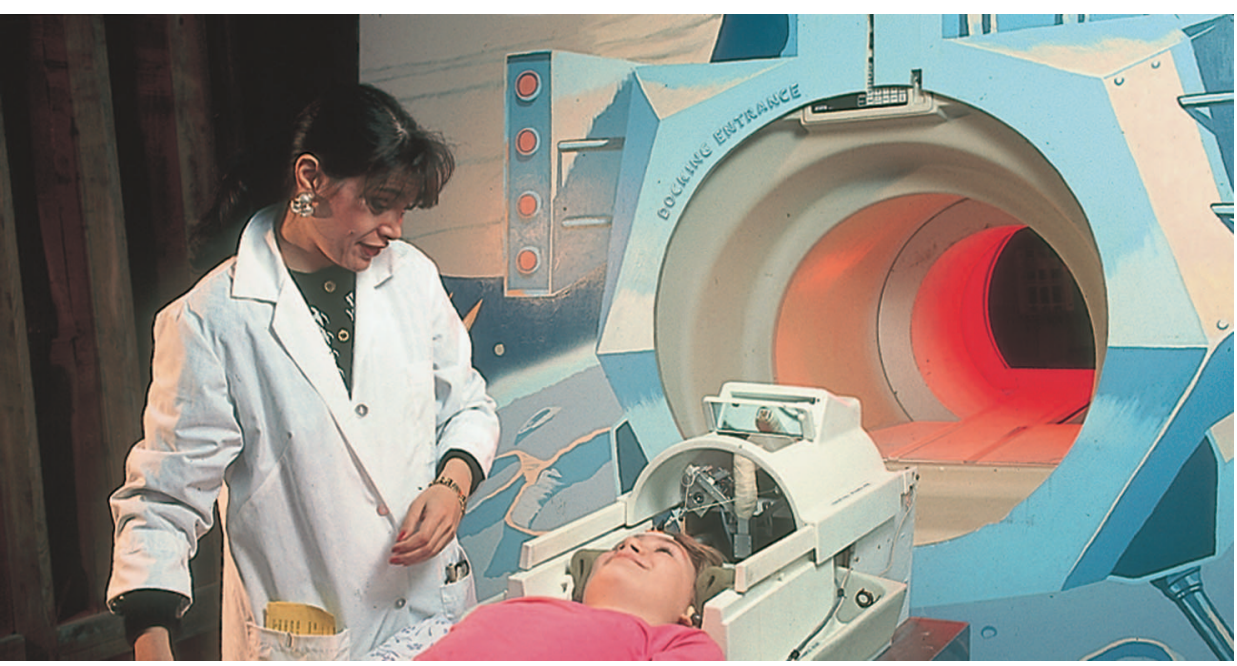

T $\mathrm{t}$ has been almost 15 years since the publication of the landmark National Institute of Neurological Disorders and Stroke tissue plasminogen activator (NINDS-tPA) trial. The findings of the NINDS-tPA trial soon led to Food and Drug Administration (FDA) approval for IV alteplase (tPA) in the treatment of acute ischemic stroke (AIS) that transformed the way neurologists approach this devastating disease. Unfortunately, 15 years removed from the NINDS-tPA trial, IV tPA remains the only FDA-approved drug for the treatment of AIS. Although no major clinical breakthrough has occurred in the AIS treatment front, newer trials have increased the spectrum of patients who can be treated, but failed to find better lytic drugs or ways to identify treatable patients

Tzu-Ching Wu, MD James C. Grotta, MD

Address correspondence and reprint requests to Dr. James C. Grotta, Department of Neurology, The University of Texas-Houston Medical School, 6431 Fannin Street, Houston, TX 77030

james.c.grotta@uth.tmc.edu

Neurology ${ }^{\circledR}$ Clinical Practice 2010;75(Suppl 1):S16-S21 using advanced imaging. Major advancements have transpired in the arena of stroke prevention, especially in endovascular therapy and management of atrial fibrillation (AF). This article aims to summarize 5 new topics in stroke treatment, prevention, and poststroke care that have or will soon affect clinical treatment of stroke patients, and to offer critiques and commentary on how the results of the trials presented can be applied to the care of individual stroke patients.

CAROTID STENTING VS ENDARTERECTOMY The relationship between carotid stenosis and increased stroke risk has been well-established and described. Results of the 3 prospective carotid endarterectomy (CEA) trials, North American Symptomatic Carotid Endarterectomy Trial, European Carotid Surgery Trial, and the Veteran's Affairs Cooperative Study Program, showed that symptomatic patients with high-grade stenosis benefited from surgery ${ }^{1}$ and established CEA as an effective treatment. In the Asymptomatic Carotid Surgery Trial, investigators found that CEA also prevented strokes in asymptomatic patients with carotid stenosis greater than $70 \%$ and younger than age $75 .{ }^{2} \mathrm{CEA}$ soon became the gold standard secondary stroke prevention treatment for carotid artery atherosclerosis. With the advent of improved angiographic procedures and devices, physicians soon began to seek nonsurgical methods to treat the condition.

The Carotid Revascularization Endarterectomy vs Stenting Trial (CREST) was recently completed and published. ${ }^{3}$ The aim of the trial was to compare outcomes of patients with either symptomatic or asymptomatic extracranial stenosis who underwent traditional endarterectomy vs carotid artery stenting (CAS) with distal protection. Results of 2,502 patients over 117 carefully selected centers in the United States and Canada were available for analysis for the study. The primary endpoint was the composite of any stroke, myocardial infarction (MI), or death during the periprocedural period or ipsilateral stroke within 4 years after randomization.

The results showed that there was no significant difference in the 4-year rate of primary endpoints achieved between the 2 procedures with 7.2\% for CAS and 6.8\% for CEA (hazard ratio [HR] for stenting 1.11; 95\% confidence interval [CI] $0.81-1.51 ; p=0.51$ ). Primary endpoints were also similar during the periprocedural period: $5.2 \%$ for CAS and $4.5 \%$ for CEA (HR for stenting 1.18 ; 95\% CI 0.82-1.68; $p=0.38$ ). Interestingly, the investigators did find that during the periprocedural period, the incidence of MI was more prevalent in the CEA population $(2.5 \%$ vs $1.1 \% ; p=0.03)$ but periprocedural strokes were more common in patients who

From the Department of Neurology, University of Texas-Houston Medical School, Houston.

Disclosure: Author disclosures are provided at the end of the article. 
underwent CAS ( $4.1 \%$ vs $2.3 \%$; $p=0.01$ ). Secondary analysis aimed to differentiate efficacy by symptomatic status, age, and sex. There was no effect of symptomatic status or sex on the primary endpoint, but the data suggest that those younger than 70 years had greater benefit from CAS, with CEA showing greater efficacy in patients older than 70 years of age.

\section{Major advancements have transpired in the arena of stroke prevention, especially in endovascular therapy and management of atrial fibrillation}

Not surprisingly, the issue of CEA vs CAS and the interpretation of the CREST results are still debated within the neurovascular community. Although the results showed that there were no differences between the procedures in achieving the primary outcome (any stroke, MI, death), it is important to highlight that stroke, which one can argue has a stronger effect on quality of life than a nonfatal MI, was more likely after CAS. The increased risk of ischemic events with CAS was also observed in the International Carotid Stenting Study trial: 7.7\% for CAS and $4.1 \%$ for CEA (HR for stenting 1.92; $95 \%$ CI 1.27-2.89; $p=0.002)^{4}$ and a substudy of the International Carotid Stenting Study trial using MRI-DWI showed 3 times more patients in the CAS group than in the CEA group had new ischemic lesions on posttreatment MRI scans. ${ }^{5}$

While the CREST trial has shown that both procedures can be done safely and are durable, at least up to 4 years, it is important to underscore that the procedures in the trial were performed by highly qualified interventionists or surgeons, which may not be available at all centers. In addition, there are other factors that may also influence treatment selection, such as location of the stenotic lesion and vessel accessibility. CREST has reinforced the robust utility of CEA and has also armed clinicians with another option to treat carotid disease, but we still need to individualize therapy to balance all risks and benefits. Clinicians have to take into account a multitude of clinical factors and also be familiar with the technical expertise of the local surgeon or interventionist in order to develop the best treatment strategy for each patient. At present, we tend to favor CAS in younger patients without extensive atherosclerosis and noncompliant vessels, and also in high surgical risk patients with bilateral disease (who are currently candidates for the ongoing Stenting and Angioplasty with Protection in Patients at High Risk for Endar- terectomy trial). We recommend CEA in most other patients.

NEW ORAL THERAPY FOR ATRIAL FIBRILLATION It has been estimated that over 2 million Americans are affected by AF and more that 75,000 strokes each year can be attributed to AF. ${ }^{6}$ Numerous trials have demonstrated the efficacy of warfarin in the treatment of AF for stroke prevention and it remains the mainstay therapy for this disease. Although efficacious, warfarin nevertheless has disadvantages that often limit its use in AF patients. Notably, warfarin causes major bleeding in $1.3 \%$ annually ${ }^{1}$ and with the multitude of drug and food interactions and narrow therapeutic window, continuous close INR monitoring is required.

The Randomized Evaluation of Long-Term Anticoagulation Therapy trial $^{6}$ was a noninferiority trial that aimed to determine the incidence of systemic embolism in patients treated with warfarin or the new oral thrombin inhibitor, dabigatran. AF patients with risks for stroke were randomized to either 110 mg or $150 \mathrm{mg}$ of dabigatran twice daily or adjusted dose warfarin (target INR 2.0 to 3.0). The primary clinical outcome was stroke or systemic embolism and the primary safety outcome was major bleeding defined as a reduction in the hemoglobin level of at least $20 \mathrm{~g} / \mathrm{L}$, transfusion of at least 2 units of blood, or symptomatic bleeding in a critical area or organ. ${ }^{6}$

In total, 18,113 patients were enrolled with a mean age of 71 years and the median follow-up was 2 years. Results showed that the primary clinical outcome was achieved in $1.53 \% /$ year of patients receiving $110 \mathrm{mg}$ of dabigatran, $1.11 \%$ /year for $150 \mathrm{mg}$ of dabigatran, and $1.69 \%$ /year for warfarin, with both doses of dabigatran demonstrating noninferiority to warfarin $(p<0.001)$. High-dose dabigatran proved to be superior to warfarin (RR 0.66; 95\% CI $0.74-$ $0.82 ; p<0.001)$ in regards to the primary endpoint. The occurrence of hemorrhagic stroke was rare in all 3 groups but the rates were lower in the dabigatran group, which achieved statistical significance (0.38\%/year warfarin, $0.12 \%$ /year $110 \mathrm{mg}$ dabigatran, $0.10 \%$ /year $150 \mathrm{mg}$ dabigatran).

Major bleeding was observed in 3.36\%/year of warfarin-treated patients, as compared to $2.71 \%$ /year (RR 0.80; 95\% CI 0.69-0.93; $p=0.003$ ) in those treated with $110 \mathrm{mg}$ dabigatran and $3.11 \%$ /year (RR 0.93 ; $95 \%$ CI $0.81-1.7 ; p=0.31)$ in the $150 \mathrm{mg}$ dabigatran group. Overall, the combined outcome including major vascular events, major bleeding, and death was $7.64 \% /$ year in the warfarin group, 7.09\%/year (RR 0.92; 95\% CI 0.84-1.02; $p=$ 0.10 ) with $110 \mathrm{mg}$ of dabigatran, and $6.91 \% /$ year 
(RR $0.91 ; 95 \%$ CI $0.82-1.00 ; p=0.04)$ with 150 mg of dabigatran.

Clinical outcomes were comparable between all doses of dabigatran and warfarin, with the high-dose dabigatran outperforming warfarin. On the other hand, the low-dose dabigatran had lower major bleeding rates than warfarin, and similar major bleeding risks were observed between warfarin and high-dose dabigatran. Currently, dabigatran is not available in the United States but indications are that it will soon be reviewed by the FDA. The cost of dabigatran, which is estimated to be 5-10 times the cost of warfarin, may be a major barrier to its widespread use if approved by the FDA. However, the lack of mandatory INR monitoring and the potential of increasing medication compliance along with its superior efficacy and less risk would prevent more disabling strokes. The increased cost of dabigatran would be outweighed by a reduction in the longterm costs of stroke care. The results of this study are encouraging, and a safer and more effective (and cost-effective) treatment may soon be available for secondary stroke prevention in AF. We anticipate using the higher $150 \mathrm{mg}$ dose in most patients, and the lower $110 \mathrm{mg}$ dose in patients with higher bleeding risk.

EXTENDING THROMBOLYSIS BEYOND THE 3-HOUR TIME WINDOW Results from the 1995 NINDS-tPA trial showed that IV tPA initiated in patients within 3 hours of stroke onset was 30\% more likely to achieve little or no disability up to a year after treatment compared to placebo. Despite the robust results of the NINDS-tPA trial, it is estimated that only $2 \%-5 \%$ of all stroke patients are treated with IV tPA nationally, ${ }^{7,8}$ largely due to delay in presentation to emergency departments. Subsequent analysis of the NINDS-tPA trial and 6 other prospective randomized tPA trials revealed that IV tPA may still be efficacious beyond 3 hours and up to 4.5 hours without unacceptable risk of hemorrhage or death.

The European Cooperative Acute Stroke Study III (ECASS III) was a randomized, placebocontrolled, phase 3 trial that aimed to assess the efficacy of IV tPA administered between 3 and 4.5 hours after stroke onset. ${ }^{9}$ Patients aged $18-80$ years, with NIH Stroke Scale (NIHSS) score $\leq 25$, with clinical diagnosis of AIS and able to receive the study drug within 3 to 4.5 hours were eligible for the trial. Major exclusion criteria included a combination of previous stroke and diabetes, severe strokes, and patients receiving warfarin even with normal INR. In total, 821 patients were enrolled and included in the intention-to-treat analysis with 418 assigned to tPA and 403 assigned to placebo.
The primary endpoint was 90-day disability measured by the modified Rankin score (mRS), dichotomized as favorable outcome ( 0 or 1 ) or unfavorable outcome (2 to 6). Secondary endpoint was a global outcome measure comprised of the composite of 90day mRS of 0 or 1 , Barthel Index $\geq 95$, and NIHSS and Glasgow Outcome Score $=0$ or 1. Safety endpoints included mortality at 90 days, any intracranial hemorrhage, symptomatic intracranial hemorrhage (sICH), and symptomatic edema.

Demographics and baseline characteristics were fairly similar between the study group and the placebo arm, except that the initial NIHSS score was slightly higher in the placebo group (median of $9 \mathrm{tPA}$ and 10 placebo; $p=0.03$ ) and the history of stroke was more prevalent in the control group (14.1\% placebo vs $7.7 \%$ tPA; $p=0.03$ ). After adjustment for these confounding variables, treatment with $\mathrm{tPA}$ was associated with an increase in favorable outcome (odds ratio 1.42; 95\% CI 1.02-1.98; $p=0.04$ ). Both intracranial hemorrhage (17.6\% placebo vs $27 \% \mathrm{tPA}, p=0.001)$ and the incidence of $\mathrm{sICH}$ $(0.3 \%$ placebo vs $2.4 \%$ tPA, $p=0.008)$ were more common in the tPA group.

In contrast to the NINDS-tPA trial, ECASS III excluded patients older than 80, those with NIHSS $>25$, patients taking oral anticoagulants, and patients with history of previous stroke and diabetes mellitus. The definition of sICH also differed between the 2 trials. In the NINDS-tPA trial, sICH was defined as a hemorrhage not seen on a previous CT scan causing any decline in neurologic status. ECASS III had a narrower definition of sICH, defined as a hemorrhage causing a decline in neurologic status by increasing the NIHSS by greater than 4 points or leading to death. Despite the differences in trial inclusion and exclusion criteria, IV tPA as compared to placebo given in the 3- to 4.5-hour window provided significantly improved clinical outcomes with acceptable risks of sICH and will no doubt change clinical practice. This extension of the time window can potentially increase the number of patients eligible for IV thrombolytics. However, since the more severe the stroke the earlier the patient presents to the emergency department, it is not clear how much the results of ECASS III will increase the number of stroke patients receiving tPA. Also, while effective out to 4.5 hours, it is important to underscore the importance of rapid treatment of AIS as it has been shown that clinical recovery is much more likely if treatment occurs within 2 hours of symptom onset. In 2008, the American Heart Association/ American Stroke Association released a science advisory recommending IV tPA in the 3- to 4.5-hour time window for eligible AIS patients, but the FDA 
has not yet extended the label indication. In the meantime, most major stroke centers, including our own, have incorporated the ECASS III results into clinical practice but obtain informed consent of the off-label use of the drug in all patients treated beyond 3 hours.

PENUMBRAL IMAGING AND NEW THROMBOLYTICS Many stroke experts believe that the "time = brain" concept for treating stroke is too simplistic, and that in many patients salvageable penumbral tissue may persist for many hours. Over the last 10 years, the use of perfusion imaging to identify such penumbral tissue in AIS has been an extremely active area of research. With the ability to identify salvageable tissue regardless of the time window, perfusion imaging can potentially improve patient selection and expand those eligible for thrombolysis. ${ }^{10}$ Many clinical trials and stroke centers have incorporated penumbral imaging into their protocols despite the lack of consensus on the appropriate way to image the ischemic penumbra and the role for its use in patient selection. ${ }^{11}$ One such recently completed trial was Desmoteplase In Acute Ischemic Stroke 2 (DIAS-2).

The DIAS-2 trial was a randomized, placebocontrolled, double-blind, dose-ranging study using perfusion imaging (MRI or CT) for patient selection to evaluate the efficacy of the highly fibrin-specific plasminogen activator desmoteplase in AIS patients treated in the 3- to 9-hour time window. Patients aged 18-85 years, with NIHSS 4-24, who had potentially salvageable tissue (estimated as at least 20\% more tissue with significantly reduced perfusion compared with the core of acute ischemic injury) 3-9 hours after symptom onset were eligible for the trial. ${ }^{12}$ Treatments were allocated on a 1:1:1 ratio with patients receiving either $90 \mu \mathrm{g} / \mathrm{kg}$ or $125 \mu \mathrm{g} / \mathrm{kg}$ of desmoteplase or placebo. In total, 193 patients were randomized, with 186 receiving treatment (57 received $90 \mu \mathrm{g} / \mathrm{kg}$ of desmoteplase, 64 received 125 $\mu \mathrm{g} / \mathrm{kg}$ of desmoteplase, 63 received placebo).

The primary efficacy endpoint was a composite 90-day outcome of improvement of NIHSS of 8 points or more from baseline or NIHSS $\leq 1$, mRS of $0-2$, and Barthel Index of 75-100. Secondary endpoints were change in infarct volume from baseline to 30 days and individual components of the 90-day endpoints. The primary safety endpoints were symptomatic ICH defined as hemorrhage confirmed on imaging that led to an increase of 4 points or more in NIHSS at 72 hours, and 90-day mortality.

Demographics and baseline characteristics were similar between the 2 study groups and the placebo arm, with median NIHSS of 9 in all 3 arms, and comparable time to treatment. Clinical composite outcome at 90 days among the 3 treatment arms was not statistically different (composite responder rate: $90 \mu \mathrm{g} / \mathrm{kg}$ desmoteplase 47\%; $125 \mu \mathrm{g} / \mathrm{kg}$ desmoteplase $36 \%$; placebo $46 \%$; $p=0.47$ ). Five sICH were observed in the study, all in the desmoteplase arms, with 2 in the $90 \mu \mathrm{g} / \mathrm{kg}$ group and 3 in the $125 \mu \mathrm{g} / \mathrm{kg}$ group. Mortality at 90 days was higher in the highdose desmoteplase arm with 14 deaths (21\%) in the $125 \mu \mathrm{g} / \mathrm{kg}$ group, 3 deaths (5\%) in the $90 \mu \mathrm{g} / \mathrm{kg}$ group, and 4 deaths $(6 \%)$ in the placebo arm. Hemorrhage did not account for most of the deaths.

Unlike the earlier DIAS-1 and Dose Escalation of Desmoteplase in Acute Stroke (DEDAS) studies using similar image-based patient selection, DIAS-2 failed to show a favorable effect of desmoteplase given 3-9 hours after stroke onset. DIAS-2, however, did differ from the preceding 2 studies in that baseline NIHSS scores were lower ${ }^{9}$ compared to a NIHSS of 12 for the DIAS and DEDAS studies. Consequently, placebo patients did much better than expected, and many patients who were enrolled probably did not have major arterial occlusion that would be advantageously treated by a lytic drug. Very importantly, the DIAS-2 investigators found that even though patients in DIAS-2 had higher mismatch volumes than patients in DIAS-1 or DEDAS, it did not translate into any noticeable benefit, bringing into question the predictive value of "mismatch" imaging. An ongoing follow-up study of desmoteplase has abandoned this image-based approach, and it is fair to say that a "tissue-based" approach as determined by imaging has yet to be proven superior than the "time-based" approach proven effective in the various tPA studies.

Another study also failed to show the benefit of a newer alternative to tPA. Tenecteplase (TNK), a modified version of $\mathrm{tPA}$, is more fibrin-specific, with a longer half-life, allowing it to be given as a bolus instead of a 1-hour infusion like tPA. ${ }^{13}$ Already approved for use in $\mathrm{MI}$ and observed to have less bleeding complications than tPA, TNK is a potential alternative to tPA for AIS as it is easier and possibly safer to administer. Prior TNK dose escalation studies showed similar clinical responses at 3 months when compared to tPA-treated patients; hence investigators moved onward and designed a phase IIB/III trial comparing IV TNK vs standard IV tPA in AIS patients within 3 hours of stroke onset hoping to find that it is a safer and at least as effective alternative.

This was a phase IIB/III multicenter, randomized, double-blind, controlled trial, and did not use any imaging selection other than plain head CT. The aims of the phase IIB study were to first establish the best dose of TNK to carry forward using an early clinical outcome measure and to provide data regard- 
ing the promise or futility of further testing of TNK vs tPA. Early clinical outcome was scored at 24 hours using a composite measure that balanced major neurologic improvement, defined as a $\geq 8$-point improvement on the NIHSS or a score of 0 at 24 hours against the risk of sICH.

In total, 112 patients were randomized into the prematurely terminated trial, with 31 patients randomized to $0.1 \mathrm{mg} / \mathrm{kg}$ TNK, 31 patients randomized to $0.25 \mathrm{mg} / \mathrm{kg}$ TNK, 19 patients randomized to 0.4 $\mathrm{mg} / \mathrm{kg}$ TNK, and 31 patients randomized to 0.9 $\mathrm{mg} / \mathrm{kg}$ tPA. The $0.4 \mathrm{mg} / \mathrm{kg}$ TNK dose was discarded as inferior after only 73 patients were randomized, and the trial was terminated due to slow enrollment after 112 patients had been randomized. Analysis showed no statistical difference in the 3-month outcomes between the 2 remaining TNK doses and tPA, with insufficient data to confidently make any conclusion about efficacy or futility.

Despite attempts of recent trials like DIAS-2 and the TNK study to demonstrate superiority of newer lytics over $\mathrm{tPA}$, tPA remains the only proven effective thrombolytic. The value of penumbral imaging has not yet been proven, with many controversies surrounding standardization of perfusion thresholding and imaging/postprocessing techniques. ${ }^{11}$ Until another proven therapy comes along, and we learn how to select patients who may qualify at later time windows, the focus of the stroke community should be on increasing the number of patients treated early with tPA, educating clinicians, emergency medical services, and hospitals to improve administration rates, and finding ways to amplify tPA when given in the ultra-early time frame.

ADVANCES IN POSTSTROKE CARE Hyperglycemia and its association with poor stroke outcome has been reported in animal models and in humans. ${ }^{14}$ However, evidence is lacking regarding the therapeutic benefits of tight glycemic control in the AIS population. The Glucose Regulation in Acute Stroke Patients (GRASP) trial was a prospective randomized 3 -arm trial that aimed to assess the safety and feasibility of tight glycemic control using 2 insulin infusion protocols in AIS patients. Enrolled patients were randomized into a tight control arm (target glucose $70-100 \mathrm{mg} / \mathrm{dL}$ ), loose control arm (target glucose $70-200 \mathrm{mg} / \mathrm{dL}$ ), or usual care control arm (target glucose $70-300 \mathrm{mg} / \mathrm{dL}$ ).

A total of 74 patients were enrolled, with 25 patients randomized into the usual care arm, $25 \mathrm{pa}$ tients randomized into the loose control arm, and 24 patients randomized into the tight control arm. The primary endpoints were safety, determined by hypoglycemia (blood glucose $<55 \mathrm{mg} / \mathrm{dL}$ ), symptomatic hypoglycemia, and feasibility determined by intarget success at 24 hours.

Results showed that hypoglycemia was more common in the tight control arm, with $30 \%$ of the patients experiencing at least 1 episode compared to only $4 \%$ in the loose and usual care arms. Symptomatic hypoglycemia was detected in only 1 patient (from the loose control arm). The median glucose in the tight control arm during the study period was $111 \mathrm{mg} / \mathrm{dL}$, with $44 \%$ meeting glucose target at 24 hours compared to the loose control arm, with a median glucose of $151 \mathrm{mg} / \mathrm{dL}$ and $92 \%$ meeting glucose target at 24 hours. In the usual care arm, 88\% met target glucose with a median glucose of $151 \mathrm{mg} / \mathrm{dL}$. Designed as a pilot safety and feasibility study, the study was not powered to detect clinical benefit between the treatment arms.

Although nonsignificant and requiring further study, GRASP casts doubt on the benefit and safety of tight glycemic control in AIS, and underscores again that stroke patients are a unique population, and that therapeutics cannot be generalized from another organ system to cerebrovascular disease.

CONCLUSION Stroke treatment and prevention can make a major difference in the effect of stroke on the individual patient and on societal costs. Within the last 2 years, a number of trials summarized here have or may soon lead to changes in clinical practice. It is important not only to continue to strive to find more effective treatment and prevention strategies for ischemic stroke, but also to work to accomplish the systematic changes to help implement these strategies.

\section{DISCLOSURE}

Dr. Wu receives a Training Grant from the NIH (5T32 NS007412-12), Dr. Grotta serves on a scientific advisory board for Lundbeck, Inc.; serves on the editorial board of the International Journal of Stroke and Stroke; holds patents re: A composition and method for treatment of cerebral ischemia; receives royalties from the publication of Acute Stroke Care: A Manual from the University of Texas-Houston Stroke Team (Cambridge, 2007) and Stroke: Pathophysiology, Diagnosis, and Management (Churchill Livingstone, 2004); and receives research support from the NIH/NINDS (P50 NS 044227 [PI], R01 NS052971 [Co-I], P01 NS046588-02 [Co-I], and T32-NS0074212-11 [PI]), the Burnett Family Stroke Fund, and the Harold Farb Research Fund.

Received June 19, 2010. Accepted in final form September 2, 2010.

\section{REFERENCES}

1. Sacco RL, Adams R, Albers G, et al. Guidelines for prevention of stroke in patients with ischemic stroke or transient ischemic attack: a statement for healthcare professionals from the American Heart Association/American Stroke Association Council on Stroke: co-sponsored by the Council on Cardiovascular Radiology and Intervention: the American Academy of Neurology affirms the value of this guideline. Stroke 2006;37:577-617. 
2. Halliday A, Mansfield A, Marro J, et al. Prevention of disabling and fatal strokes by successful carotid endarterectomy in patients without recent neurological symptoms: randomised controlled trial. Lancet 2004;363:1491-1502.

3. Brott TG, Hobson RW 2nd, Howard G, et al. Stenting versus endarterectomy for treatment of carotid-artery stenosis. N Engl J Med;363:11-23.

4. Ederle J, Dobson J, Featherstone RL, et al. Carotid artery stenting compared with endarterectomy in patients with symptomatic carotid stenosis (International Carotid Stenting Study): an interim analysis of a randomised controlled trial. Lancet;375:985-997.

5. Bonati LH, Jongen LM, Haller S, et al. New ischaemic brain lesions on MRI after stenting or endarterectomy for symptomatic carotid stenosis: a substudy of the International Carotid Stenting Study (ICSS). Lancet Neurol;9:353-362.

6. Connolly SJ, Ezekowitz MD, Yusuf S, et al. Dabigatran versus warfarin in patients with atrial fibrillation. N Engl J Med 2009;361:1139-1151.

7. Albers GW, Olivot JM. Intravenous alteplase for ischaemic stroke. Lancet 2007;369:249-250.

8. Katzan IL, Hammer MD, Hixson ED, Furlan AJ, AbouChebl A, Nadzam DM. Utilization of intravenous tissue plasminogen activator for acute ischemic stroke. Arch Neurol 2004;61:346-350.

9. Hacke W, Kaste M, Bluhmki E, et al. Thrombolysis with alteplase 3 to 4.5 hours after acute ischemic stroke. N Engl J Med 2008;359:1317-1329.

10. Parsons MW. Perfusion CT: is it clinically useful? Int J Stroke 2008;3:41-50.

11. Wintermark M, Albers GW, Alexandrov AV, et al. Acute stroke imaging research roadmap. Stroke 2008;39:16211628.

12. Hacke W, Furlan AJ, Al-Rawi Y, et al. Intravenous desmoteplase in patients with acute ischaemic stroke selected by MRI perfusion-diffusion weighted imaging or perfusion CT (DIAS-2): a prospective, randomised, double-blind, placebocontrolled study. Lancet Neurol 2009;8:141-150.

13. Haley EC Jr, Thompson JL, Grotta JC, et al. Phase IIB/III trial of tenecteplase in acute ischemic stroke: results of a prematurely terminated randomized clinical trial. Stroke 2010;41:707-711.

14. Parsons MW, Barber PA, Desmond PM, et al. Acute hyperglycemia adversely affects stroke outcome: a magnetic resonance imaging and spectroscopy study. Ann Neurol 2002;52:20-28.

\section{If you liked this article, you may be interested in. .}

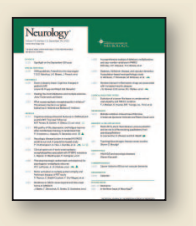

\section{Neurology}

Schellinger et al. Evidence-based guideline: The role of diffusion and perfusion MRI for the diagnosis of acute ischemic stroke: Report of the Therapeutics and Technology Assessment Subcommittee of the American Academy of Neurology. July 13, 2010; www.neurology.org

Dhamoon et al. Quality of life declines after first ischemic stroke: The Northern Manhattan Study. July 27, 2010; www.neurology.org

Smith et al. Do all ischemic stroke subtypes benefit from organized inpatient stroke care? August 3, 2010; www.neurology.org

Ovbiagele et al. Disability status at 1 month is a reliable proxy for final ischemic stroke outcome. August 24, 2010; www.neurology.org

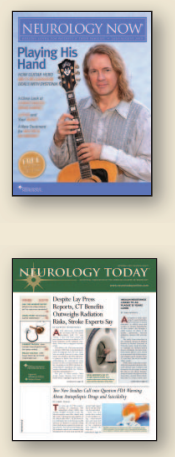

\section{Neurology Now}

Seemant Chaturvedi. Ask the Experts: Stroke and depression. Jan/Feb 2010; www.neurologynow.org

Amy Paturel. Walk This Way: Treadmill and task-oriented training mobilize people with stroke, Parkinson's, and other neurologic diseases that impact gait. May/June 2010; www.neurologynow.org

\section{Neurology Today}

Charlene Laino. Cilostazol Beats Out Aspirin for Secondary Stroke Prevention. April 1, 2010; www.neurotodayonline.com

Ed Susman. 'Drip-and-Ship' Appears as Safe as In-hospital Stroke Treatment. April 15, 2010; www.neurotodayonline.com

Kurt Samson. Study Suggests tPA is 'Safe' for Ischemic Stroke False Alarms. May 20, 2010; www.neurotodayonline.com

Kurt Samson. New AAN Guidelines on Stroke Imaging. Aug. 5, 2010; www.neurotodayonline.com

Kierstin Wesolowski. Despite Lay Press Reports, CT Benefits Outweigh Radiation Risks, Stroke Experts Say. Sept. 2, 2010; www.neurotodayonline.com 


\section{Neurology}

\section{Stroke treatment and prevention: Five new things}

Tzu-Ching Wu and James C. Grotta

Neurology 2010;75;S16-S21

DOI 10.1212/WNL.0b013e3181fb3616

\section{This information is current as of November 1,2010}

\section{Updated Information \&} Services

References

Subspecialty Collections

Permissions \& Licensing

Reprints including high resolution figures, can be found at: http://n.neurology.org/content/75/18_Supplement_1/S16.full

This article cites 11 articles, 3 of which you can access for free at: http://n.neurology.org/content/75/18_Supplement_1/S16.full\#ref-list-1

This article, along with others on similar topics, appears in the following collection(s):

\section{All Cerebrovascular disease/Stroke}

http://n.neurology.org/cgi/collection/all_cerebrovascular_disease_strok $\mathrm{e}$

Information about reproducing this article in parts (figures,tables) or in its entirety can be found online at:

http://www.neurology.org/about/about_the_journal\#permissions

Information about ordering reprints can be found online:

http://n.neurology.org/subscribers/advertise

Neurology ${ }^{\circledR}$ is the official journal of the American Academy of Neurology. Published continuously since 1951, it is now a weekly with 48 issues per year. Copyright Copyright (? 2010 by AAN Enterprises, Inc.. All rights reserved. Print ISSN: 0028-3878. Online ISSN: 1526-632X.

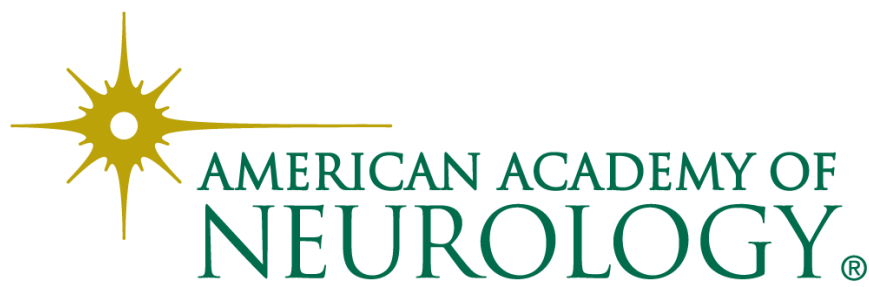

